\title{
RESEARCH
}

Open Access

\section{Deep sequencing analysis of toad Rhinella schneideri skin glands and partial biochemical characterization of its cutaneous secretion}

Priscila Yumi Tanaka Shibao ${ }^{1}$, Camila Takeno Cologna', Romualdo Morandi-Filho², Gisele Adriano Wiezel', Patricia Tiemi Fujimura ${ }^{2}$, Carlos Ueira-Vieira ${ }^{2}$ and Eliane Candiani Arantes ${ }^{2,3^{*}}$ (iD

\begin{abstract}
Background: Animal poisons and venoms are sources of biomolecules naturally selected. Rhinella schneideri toads are widespread in the whole Brazilian territory and they have poison glands and mucous gland. Recently, protein from toads' secretion has gaining attention. Frog skin is widely known to present great number of host defense peptides and we hypothesize toads present them as well. In this study, we used a RNA-seq analysis from $R$. schneideri skin and biochemical tests with the gland secretion to unravel its protein molecules.

Methods: Total RNA from the toad skin was extracted using TRizol reagent, sequenced in duplicate using Illumina Hiseq2500 in paired end analysis. The raw reads were trimmed and de novo assembled using Trinity. The resulting sequences were submitted to functional annotation against non-redundant NCBI database and Database of Anuran Defense Peptide. Furthermore, we performed caseinolytic activity test to assess the presence of serine and metalloproteases in skin secretion and it was fractionated by fast liquid protein chromatography using a reverse-phase column. The fractions were partially sequenced by Edman's degradation.

Results: We were able to identify several classes of antimicrobial peptides, such as buforins, peroniins and brevinins, as well as PLA ${ }_{2}$ lectins and galectins, combining protein sequencing and RNA-seq analysis for the first time. In addition, we could isolate a PLA from the skin secretion and infer the presence of serine proteases in cutaneous secretion.

Conclusions: We identified novel toxins and proteins from R. schneideri mucous glands. Besides, this is a pioneer study that presented the in depth characterization of protein molecules richness from this toad secretion. The results obtained herein showed evidence of novel AMP and enzymes that need to be further explored.
\end{abstract}

Keywords: RNA-seq, Rhinella schneideri, Toad secretion, Transcriptome, Illumina, Cutaneous secretion, Skin secretion, Toad protein

\footnotetext{
* Correspondence: ecabraga@fcfrp.usp.br

2Laboratory of genetics - LABGEN, Institute of Genetics and Biochemistry,

Campus Umuarama, Federal University of Uberlândia, Avenida Pará,

Uberlândia, MG 1720, Brazil

${ }^{3}$ Department of Physics and Chemistry, University of São Paulo, School of

Pharmaceutical Sciences of Ribeirão Preto, Av. do Café s $/ n^{\circ}$, Monte Alegre,

Ribeirão Preto, SP 14040-903, Brazil

Full list of author information is available at the end of the article
}

(c) The Author(s). 2018 Open Access This article is distributed under the terms of the Creative Commons Attribution 4.0 International License (http://creativecommons.org/licenses/by/4.0/), which permits unrestricted use, distribution, and reproduction in any medium, provided you give appropriate credit to the original author(s) and the source, provide a link to the Creative Commons license, and indicate if changes were made. The Creative Commons Public Domain Dedication waiver (http://creativecommons.org/publicdomain/zero/1.0/) applies to the data made available in this article, unless otherwise stated. 


\section{Background}

Animal's and microorganisms' secretions, as well as plant extracts, have been used as folk medicine since the dawn of the humanity [1].Therefore, molecules found in poisons and venoms are interesting, once they were selected by evolution to act in their molecular targets with high specificity $[1,2]$. Such molecules can be used for feeding (predation), defense or even to dispose advantage in inter and intra-specific competition [3, 4]. As an example, the glandular secretory product from toads Bufo melanostictus Schneider and Bufo Bufo gargarizans cantor, known as Chan $\mathrm{Su}$, is used as medicine in treatment for several physiological disturbances [5].

Rhinella schneideri toads are widely found in South America territory: Paraguay, Bolivia, Argentina, Uruguay and Brazil. Regarding the Brazilian territory, they are found especially in cerrado. These toads have shown remarkable adaptations skills and live in urban areas as well as rural [6].

These toads present two types of glands: granulous or parotoid and mucous glands. The first one is responsible for the animal protection against predators and are located in the postorbital region of the animal's body; they can look bigger when the animal feels endangered due to body's inflation, and acts as airbags against predator bites $[7,8]$. The secretions are composed mainly by biogenic amines and steroids, as bufodienolides and bufotoxines, but they also produce proteins and glicoconjugate molecules $[9,10]$. Although it was previously believed that this poison presented only few or even no protein, lately it was revealed this secretion possess up to $30 \%$ of weight in proteins, but there is a lack of data to full assess them $[11,12]$. Rhinella schneideri parotoid gland poison has shown activity against different human cancer cells proliferation [13], activate human complement system [14] and inhibit chymotrypsin [15]. Protein components present anti-inflammatory, anti-nociceptive and toxic activities in mice [16] Similarly, protein from Bufo bufo parotoid secretions are likely to play a role in cardiotoxic effects [17] and B. bufo, B. verrucosissimus and Bufotes variabilis both parotoid and skin secretion protein are capable of inhibiting different gram-negative bacteria and cytotoxic effects on different types of cells [18].

Anuran cutaneous gland secretions are widely known to contain several classes of antimicrobial peptides (AMP) and function as the first barrier against microorganisms. Although frogs' mucous gland secretions are broadly studied and there are more than 40 classes of AMP reported up to date [19], there is no survey regarding $R$. schneideri putative protein and AMPs in the cutaneous secretions. The "omics" technologies are powerful tools to overcome this issue.
Transcriptomics, one of the "omics techniques", is one powerful approach to unravel peptides and protein in a holistic manner. Currently, RNA-seq is the state-of-art technique used to predict all protein molecules that can be produced by a specific issue with the greatest outcome of information, thus making possible the discovery of minor toxins that could not be detected through traditional techniques due to their low abundance in the final secretion [20]. This approach was used to unravel frogs AMP and adaptations [21, 22] and immune system [23]. However, there is no transcriptomic information regarding toads' skin peptides and proteins.

In order to overcome this lack of information, we constructed a RNA-seq transcriptome from skin of an individual $R$. schneideri toad. The transcriptome was sequenced in duplicate using Illumina HiSeq 2500, the reads were treated and the contigs were de novo assembled with the aid of Trinity. The results were annotated against non-redundant (nr) NCBI database and enriched with database of anuran defense peptides (DADP). Thus, the cutaneous secretion from the same toad, milked prior its death, was used to carry out biochemical analysis, as assessing its protein profile by SDS-PAGE, RP-FPLC fractionation in C18 column, peptide and protein sequencing by Edman's degradation and activity upon casein to better investigate this secretion. To the best of our knowledge, this is the first study to unravel the potential of $R$. schneideri cutaneous gland secretions.

\section{Methods}

\section{Ethics statement}

Animal's experiments were designed according to the Normative Resolution N. 13, from Brazilian Minister of Science, Technology and Innovation. The experiments were reviewed and approved by the Animals usage Ethic Committee from School of Pharmaceuticals Science of Ribeirão Preto - University of São Paulo ( $\mathrm{N}^{\circ}$ : 15.1.341.60.2).

\section{Sample collection and RNA extraction}

One adult Rhinella schneideri toad was sacrificed through anesthetic overdose and the skin was dissected for mRNA assessment. According to the animal's body weight, a dose of combined anesthetic ketamine (10 $\mathrm{mg} / \mathrm{kg}$ ) and pentobarbital (150 mg/ kg) was applied intraperitoneally after 3 days of cutaneous secretion milking through electric stimulation $(5 \mathrm{~V}, 100 \mathrm{~Hz}, 140$ $\mathrm{ms})$. Toad skin patches were carefully dissected and washed with RNAlater ${ }^{\ominus}$ (Life technologies, USA), immediately thawed with dry ice and ethanol bath and kept at $-80{ }^{\circ} \mathrm{C}$ until the moment of use. All the instruments and materials used were previously cleaned in Diethylpyrocarbonate (DEPC) solution $0,1 \%(v / v)$. 
The total RNA was extracted using liquid nitrogen and TRIzol $^{\circledR}$ reagent (Life technologies) following the manufacturer's instructions. RNA integrity was assessed with $1 \%$ agarose gel and quantified with a Qubit $^{\oplus}$ RNA assay Kit with a Qubit $^{\oplus} 2.0$ Fluorometer (Life technologies). Thus, the RNA integrity was attested using 2100 Bioanalyzer (Agilent, USA) analysis.

Toad's cutaneous secretion (CS) was stored at $-20^{\circ}$ $\mathrm{C}$ until the moment of usage for RP-FPLC and biochemical analysis.

\section{Transcriptome construction and sequencing}

The transcriptome was constructed using the TruSeq Stranded mRNA library kit (Illumina, USA) according to the manufacturer's instructions. The library containing $100 \mathrm{bp}$ fragments was paired-end sequenced in duplicate in the Illumina HiSeq 2500 platform (Illumina).

\section{De novo assembling and functional annotation}

Raw reads were trimmed using FastQC $(\mathrm{Q}<20)$ [24] and the adaptor sequences were discarded. The quality control was confirmed using the FastQC tool and the reads with good quality were submitted to the de novo assembling using the Trinity software with $\mathrm{K}$-mer $=31$. The reads were mapped against the constructed transcriptome using the Tophat tool to identify splice junctions between exons. Transcripts per million (TPM) was calculated using Salmon tool. The contigs were assembled against National Center for Biotechnology Information (NCBI) non-redundant (NR) database, with the aid of FunctionAnnotator website available at http://163.25.92.60/index.php [25], and specific anuran antimicrobial peptides (AMP) database DADP [26] using the blastx algorithm, to obtain the functional annotation. The resulting annotated sequences were the ones with cut-off value of significance lower than $1 \times$ $10^{-5}$, coverage higher than $70 \%$ and protein identity (pident) higher than 60.

\section{Fractionation of cutaneous secretion (CS) by RP-FPLC, SDS-PAGE and $\mathrm{N}$-terminal sequencing}

CS was lyophilized and the dried secretion (25 mg) was dispersed in deionized water $(5 \mathrm{~mL})$. The insoluble part was separated after centrifugation $(10.000 \mathrm{x} \mathrm{g}, 5 \mathrm{~min}$, room temperature) and the supernatant was filtered in $0.22 \mu \mathrm{m}$ polyvinylidene fluoride (PVDF) membrane. CS solution $(1,5 \mathrm{~mL})$ was submitted to fast protein liquid chromatography (FPLC) in a C18 column ( $5 \mu \mathrm{m}, 250 \times$ 10.0 mm, $300 \AA$ A , Jupiter, Phenomenex) using Äkta Pure system (GE Healthcare) as described by Shibao et al. [15]. C18 column was firstly equilibrated with solution A (TFA $0,1 \%$ ) and the fractions were eluted with segmented gradient of acetonitrile until $100 \%$ of solution
B (acetonitrile $60 \%$ in TFA $0,1 \%$ ) under $5 \mathrm{~mL} / \mathrm{min}$ flow rate and $214 \mathrm{~nm}$ monitoring. The resulting fractions were collated and storage at $-20{ }^{\circ} \mathrm{C}$ until the moment of usage. The chromatographic profile was generated using the software Unicorn 5.20 (GE Healthcare).

An aliquot of $100 \mu \mathrm{L}$ of each fraction was dried and dispersed in 50\% acetonitrile (ACN) solution. Each fraction was submitted to sodium dodecyl-sulphate-polyacrylamide gel electrophoresis (SDS-PAGE), according to Schagger and Von Jagow [27]. In addition, different volumes (5, 10 and $20 \mu \mathrm{L}$ ) of the crude secretion used to RP-FPLC was also submitted to SDS-PAGE. Bench marker Amersham low molecular weight calibration kit for SDS electrophoresis (GE Healthcare) was also used to estimate protein molecular weight. The gel was submitted to $90 \mathrm{~V}, 40 \mathrm{~mA}$ and $15 \mathrm{~W}$ for $4 \mathrm{~h}$ and stained with the PlusOne Silver Staining Kit (GE Healthcare).

Protein fractions identified in the SDS-PAGE were submitted to amino terminal sequencing through Edman degradation [28] by the automatic protein sequenator model PPSQ-334 (Shimadzu).

\section{Peptides and protein alignment}

Primary peptides and proteins sequences were deduced from the cDNAs sequences from the transcriptomes with Expasy translator tool. The deduced sequences and the sequences determined by $\mathrm{N}$-terminal sequencing were aligned using Multalin algorithm [29]. The alignments were formatted using Espript 3.0 [30].

\section{Caseinolytic activity}

A chromogenic proteolytic assay with the CS was performed in the presence and absence of ethylenediamine tetraacetic acid (EDTA) and phenylmethylsulfonyl fluoride (PMSF). The assay was conducted following the method described by Wang [31]. For this assay, we used $90 \mu \mathrm{L}$ azocasein $(10 \mathrm{mg} / \mathrm{mL})$ in $50 \mathrm{mM}$ Tris- $\mathrm{HCl}$ buffer with $0.15 \mathrm{M} \mathrm{NaCl}$ and $0.15 \mathrm{M} \mathrm{CaCl}_{2}(\mathrm{pH} 8.0)$, different volumes $(10 \mu \mathrm{l}, 20 \mu \mathrm{l}$ and $30 \mu \mathrm{l})$ of CS $(5 \mathrm{mg}$ of dried secretion in $1 \mathrm{~mL}$ of deionized water), $100 \mathrm{mM}$ EDTA or $100 \mathrm{mM}$ PMSF and Tris- $\mathrm{HCl}$ buffer solution (100 mM) to complete the reactions to $120 \mu \mathrm{L}$. Positive control was performed using $10 \mu \mathrm{L}$ Trypsin $(100 \mathrm{mM})$ and negative control was carried out using the same volume of buffer. The reactions were incubated at $37^{\circ} \mathrm{C}$ for $90 \mathrm{~min}$ and stopped by adding $120 \mu \mathrm{L}$ of $0.5 \mathrm{M}$ trichloroacetic acid. All the tubes were centrifuged at $1000 \times g$ for $5 \mathrm{~min}$, $150 \mu \mathrm{L}$ of the supernatant was mixed with the same volume of $0.5 \mathrm{M} \mathrm{NaOH}$ and absorbance was determined at $450 \mathrm{~nm}$. This assay was carried out in triplicate. Data were plotted using the software GraphPad Prism 6.0 (GraphPad Software Inc). 


\section{Results}

Transcriptome sequencing, de novo assembly and functional annotation

The same transcriptome was sequenced in duplicate resulting in $129,467,414$ and $131,652,320$ raw reads (considering forward and reverse reads) for each duplicate. The data obtained from de novo assembling is summarized in Table 1 . The contigs were analyzed according to their functional annotation regarding Gene Ontology (Additional file 1), hits with deposited nucleotide and protein sequences from nr NCBI database and DADP, being the latter very important for results enrichment, once there is not much information regarding toads in NCBI database.

\section{AMP assessment}

The functional analysis of the transcriptome data and the AMP Database showed the presence of 43 different peptides and protein classes. Table 2 summarizes the more abundant contigs $(\mathrm{TPM}>100)$ and are clustered in 33 classes of AMP. The five major classes of AMP, considering the TPM values, are kassinin, temporin, peroniin, rugosauperolein and buforin.

\section{Other proteins of interest}

The main protein of interest that are not considered AMP are listed in Table 3. Two contigs related to cobatoxin were found in this study. The first one, identified as TRINITY_DN69643_c0_g1_i, is identical to cobatoxin from Helicoverpa armigera, identified by access number ADR51150.1 (gi|313,247,974). The second one, identified as TRINITY_DN121110_c0_g1_i1, has matched cobatoxin A from Spodoptera exigua (gi|827,029,657).

In the present transcriptome we found 19 full-length sequences with high homology to $\mathrm{PLA}_{2}$. Interestingly, two contigs are similar to snake $\mathrm{PLA}_{2}$. Contig TRINITY_DN72825_c0_g1_i1 encodes a PLA 2 highly similar to Crotalus durissus terrificus $\mathrm{PLA}_{2}$ crotoxin basic subunit (gi 48,429,036) (Fig. 1a). This PLA $_{2}$ was also identified in the raw skin secretion in the fractions 24A, 25 and 26 (Fig. 2) and confirmed by Edman degradation sequencing of the fractions. Contig TRINITY_DN72825_c0_g2_i1 is

Table 1 Statistical analysis of the transcriptome sequencing and de novo assembling with Trinity

\begin{tabular}{ll}
\hline Parameters & Values \\
\hline Number os trimmed reads & 174,308 \\
Average size (bp) & 633.58 \\
N50 (bp) & 365 \\
Largest sequence (bp) & 22,684 \\
Smallest sequence (bp) & 201 \\
\hline
\end{tabular}

also related to $\mathrm{PLA}_{2}$ from C. d. terrificus (Fig. 1b), but to the acid subunit (gi|129,456).

This study identified one full-length contig related to metalloproteases and 14 contigs related to serine proteases. Contig TRINITY_DN11900_c0_g1_i1 is highly homologous to a metallo-disintegrin from Xenopus laevis. Fourteen full-length sequences related to serine proteases were obtained. Six of them showed high similarity to Crotalus ssp snakes, being three (TRINITY_DN87578_c1_g1_i2, TRINITY_DN87578_c1_g1_i5, TRINITY_DN87578_c1_g1_i6,) containing the same coding sequence (herein named RsSVSP) highly related to gyroxin (Fig. 3). Contigs TRINITY_DN87578_c1_g1 _i3, TRINITY_DN87578_c1_g1_i4 and TRINITY_DN8 7578_c1_g1_i8 encode a protein (RsSVSP2) very similar to serine protease 6 from $C$. adamanteus (gi|338,855,342).

Thirteen complete open reading frames (ORFs) related to galectins and 6 related to ficolins were found in the transcriptome. From those, 12 are related to predicted galectin from different genomes and transcriptomes. Contig TRINITY_DN92920_c1_g1_i1 is similar to galectin from Rhinella arenarum ovary. Four complete ORFs were found matching different galectins from Xenopus genome assessment. All contigs related to ficolins were annotated against model organisms' genome (Хеnорus spp. and Monodelphis domestica).

\section{Fractionation of CS by RP-FPLC, SDS-PAGE and N-terminal sequencing}

Crude secretion SDS-PAGE profile is shown in Additional file 2. CS was separated in 26 fractions, named CS1 to CS26 (Fig. 2). The fractions were further submitted to SDS-PAGE, gel was stained with silver and the fractions named CS1, CS24, CS25 and CS26 (Fig. 2, insert) were identified containing protein compounds. In addition, probably fractions CS5, CS21 and CS22 also contain protein molecules, but due to its low concentration, they were not investigated in this study.

Both gels show some interference on their top, probably caused by the silver staining. It is possible to see bands with approximately $16 \mathrm{kDa}$ in the fractions CS24, CS25 and CS26, which showed similar diffusion profiles. Therefore, these fractions were submitted to $\mathrm{N}$-terminal sequencing by Edmans' degradation, but it was possible to obtain only CS1 and CS24 - CS26 partial sequences (Table 4).

\section{Caseinolytic activity}

The transcriptome functional annotation showed some sequences that can be related to serine and metalloproteases. In order to investigate if the sequences could really deduce these enzymes, we performed a proteolytic test using azocasein as substrate (Fig. 4). 
Table 2 List of main antimicrobial peptides from transcriptome

\begin{tabular}{|c|c|c|c|c|}
\hline AMP families & Accesion number & Identification & ENA Transcript identification & TPM \\
\hline Aurein & sp|P69021 & Aurein-3.1 & TRINITY_DN64440_C0_g1_i1 & 256.721 \\
\hline \multirow[t]{6}{*}{ Bombesin } & sp|P84211 & Bombesin-like peptide & TRINITY_DN48099_C0_g1_i1 & 164.379 \\
\hline & \multirow[t]{3}{*}{ sp|P84212 } & \multirow[t]{3}{*}{ Bombesin-like peptide } & TRINITY_DN27392_C0_g1_i1 & 192.298 \\
\hline & & & TRINITY_DN61842_CO_g2_i1 & 106.099 \\
\hline & & & TRINITY_DN80913_C0_g1_i2 & 102.443 \\
\hline & sp|P84214 & Bombesin & TRINITY_DN71876_C0_g1_i1 & 117.297 \\
\hline & sp|P86026 & $\begin{array}{l}\text { [Asn3,Lys6,Phe13] } \\
\text { 3-14-bombesin }\end{array}$ & TRINITY_DN76333_c0_g1_i1 & 135.698 \\
\hline Bradykinin-like peptide & sp|P84823 & Bradykinin-like peptide & TRINITY_DN71047_c0_g1_i2 & 123.521 \\
\hline \multirow[t]{2}{*}{ Brevinin } & sp|P32423 & Brevinin-1 & TRINITY_DN70354_c0_g3_i1 & 175.329 \\
\hline & sp|P82233 & Brevinin-1Ta & TRINITY_DN73322_c2_g2_i1 & 122.993 \\
\hline \multirow[t]{4}{*}{ Buforin } & \multirow[t]{4}{*}{$\mathrm{sp} \mid \mathrm{COHJB7}$} & \multirow[t]{4}{*}{ Buforin-EC } & TRINITY_DN54614_C0_g1_i1 & 766.952 \\
\hline & & & TRINITY_DN60267_c0_g1_i1 & 190.875 \\
\hline & & & TRINITY_DN60267_c1_g1_i1 & 231.457 \\
\hline & & & TRINITY_DN60267_c0_g2_i1 & 228.282 \\
\hline \multirow[t]{2}{*}{ Caerin } & \multirow[t]{2}{*}{ sp|P69032 } & \multirow[t]{2}{*}{ Caerin-2.3 } & TRINITY_DN66726_C0_g1_i1 & 182.654 \\
\hline & & & TRINITY_DN66726_C0_g1_i2 & 121.242 \\
\hline Caeridin & sp|P82076 & Caeridin-4 & TRINITY_DN79589_c4_g3_i1 & 226.024 \\
\hline Citropin & sp|P81840 & Citropin-1.2 & TRINITY_DN86384_c3_g3_i1 & 111.715 \\
\hline Cruzioseptin & sp|COHK11 & Cruzioseptin-15 & TRINITY_DN70275_c0_g1_i1 & 293.883 \\
\hline Dermaseptin & sp|P85523 & Dermaseptin-1 & TRINITY_DN76697_c0_g1_i2 & 115.602 \\
\hline Hylambatin & sp|P08614 & Hylambatin & TRINITY_DN87362_c21_g12_i1 & 174.475 \\
\hline Hylin & sp|P84003 & Hylin-b2 & TRINITY_DN84946_C0_g1_i2 & 971.000 \\
\hline Hyposin & sp|P84957 & Hyposin-HA3 & TRINITY_DN72338_c1_g1_i1 & 616.344 \\
\hline Japonicin & sp|P83306 & Japonicin-1 & TRINITY_DN64014_C0_g1_i1 & 310.058 \\
\hline \multirow[t]{5}{*}{ Kassinin } & \multirow[t]{3}{*}{ sp|P08611 } & \multirow[t]{3}{*}{ Kassinin } & TRINITY_DN120600_CO_g1_i1 & 346.986 \\
\hline & & & TRINITY_DN127618_c2_g1_i1 & 5081.92 \\
\hline & & & TRINITY_DN52216_CO_g1_i1 & 247.853 \\
\hline & \multirow[t]{2}{*}{ sp|P42988 } & \multirow[t]{2}{*}{ Kassinin-like peptide } & TRINITY_DN81065_c1_g3_i1 & 130.979 \\
\hline & & & TRINITY_DN87139_c3_g3_i1 & 164.946 \\
\hline \multirow[t]{3}{*}{ Nigrocin } & sp|B3A0M7 & Nigrocin-2JDb & TRINITY_DN87453_c9_g4_i1 & 327.271 \\
\hline & \multirow[t]{2}{*}{ sp|P0C8U1 } & \multirow[t]{2}{*}{ Nigrocin-2HSb } & TRINITY_DN85182_c2_g10_i1 & 122.641 \\
\hline & & & TRINITY_DN85182_c2_g3_i1 & 221.000 \\
\hline Peptide PGLa & sp|COHKP4 & Peptide PGLa-R6 & TRINITY_DN80328_c3_g2_i1 & 229.068 \\
\hline \multirow[t]{4}{*}{ Peroniin } & sp|P86487 & Peroniin-1.1 & TRINITY_DN53498_C0_g1_i1 & 279.654 \\
\hline & sp|P86488 & Peroniin-1.1a & TRINITY_DN67234_C0_g1_i1 & 347.803 \\
\hline & sp|P86493 & Peroniin-1.3a & TRINITY_DN81622_C0_g1_i1 & 129.528 \\
\hline & sp|P86495 & Peroniin-1.1b & TRINITY_DN46821_CO_g1_i1 & 841.554 \\
\hline Phyllocaerulein & sp|P86625 & [Arg4]-Phyllocaerulein & TRINITY_DN85513_c6_g2_i2 & 280.537 \\
\hline \multirow[t]{5}{*}{ Phylloseptin } & sp|P84569 & Phylloseptin-4 & TRINITY_DN87185_C0_g1_i2 & 109.815 \\
\hline & sp|P84931 & Phylloseptin-3 & TRINITY_DN64642_CO_g2_i1 & 168.944 \\
\hline & \multirow[t]{3}{*}{ sp|P86283 } & \multirow[t]{3}{*}{ Phylloseptin Bu-2 } & TRINITY_DN78083_CO_g4_i1 & 389.329 \\
\hline & & & TRINITY_DN78083_c0_g6_i1 & 334.985 \\
\hline & & & TRINITY_DN78083_c0_g8_i1 & 126.679 \\
\hline
\end{tabular}


Table 2 List of main antimicrobial peptides from transcriptome (Continued)

\begin{tabular}{|c|c|c|c|c|}
\hline AMP families & Accesion number & Identification & ENA Transcript identification & TPM \\
\hline \multirow[t]{2}{*}{ Ranacyclin } & \multirow[t]{2}{*}{ sp|P83663 } & \multirow[t]{2}{*}{ Ranacyclin-E } & TRINITY_DN46765_c0_g1_i1 & 685.023 \\
\hline & & & TRINITY_DN81581_C5_g1_i4 & 371.815 \\
\hline Ranalexin & sp|P82876 & Ranalexin-1Ca & TRINITY_DN110865_c1_g1_i1 & 343.086 \\
\hline Ranatensin & sp|P08951 & Ranatensin-C & TRINITY_DN70922_C0_g2_i1 & 116.215 \\
\hline Ranauterin & sp|P86020 & Ranatuerin-9 & TRINITY_DN85516_C7_g13_i1 & 111.483 \\
\hline \multirow[t]{5}{*}{ Riparin } & \multirow[t]{2}{*}{ sp|P86125 } & \multirow[t]{2}{*}{ Riparin-1.2 } & TRINITY_DN83324_C7_g3_i1 & 215.018 \\
\hline & & & TRINITY_DN92795_C0_g1_i1 & 164.545 \\
\hline & \multirow[t]{3}{*}{ sp|P86126 } & \multirow[t]{3}{*}{ Riparin-1.3 } & TRINITY_DN85374_c2_g1_i1 & 330.835 \\
\hline & & & TRINITY_DN86546_C0_g1_i2 & 264.226 \\
\hline & & & TRINITY_DN86547_c3_g6_i1 & 130.999 \\
\hline Rothein & sp|P86510 & Rothein 2.2 & TRINITY_DN87840_c8_g1_i1 & 261.426 \\
\hline \multirow[t]{3}{*}{ Rugosin } & sp|P08616 & Rugosauperolein-2 & TRINITY_DN87822_c5_g12_i1 & 826.816 \\
\hline & \multirow[t]{2}{*}{ sp|P84912 } & \multirow[t]{2}{*}{ Rugosin A-like peptide } & TRINITY_DN82274_C0_g1_i5 & 196.425 \\
\hline & & & TRINITY_DN82274_c0_g1_i6 & 314.015 \\
\hline Signiferin & sp|P86123 & Signiferin-1 & TRINITY_DN86818_c0_g1_i1 & 140.473 \\
\hline \multirow[t]{18}{*}{ Temporin } & \multirow[t]{6}{*}{$\mathrm{sp} \mid \mathrm{COHJB9}$} & \multirow[t]{6}{*}{ Temporin-ECa } & TRINITY_DN86407_c15_g1_i1 & 274.636 \\
\hline & & & TRINITY_DN86407_c15_g2_i1 & 915.026 \\
\hline & & & TRINITY_DN86407_c15_g3_i1 & 478.761 \\
\hline & & & TRINITY_DN86407_c15_g4_i1 & 527.791 \\
\hline & & & TRINITY_DN86407_c15_g5_i1 & 256.836 \\
\hline & & & TRINITY_DN86407_c15_g7_i1 & 176.844 \\
\hline & sp|P0C5X6 & Temporin-1DYa & TRINITY_DN29229_CO_g1_i1 & 133.681 \\
\hline & \multirow[t]{2}{*}{ sp|P56917 } & \multirow[t]{2}{*}{ Temporin-A } & TRINITY_DN13166_C0_g1_i1 & 527.935 \\
\hline & & & TRINITY_DN13166_C0_g2_i1 & 158.363 \\
\hline & \multirow[t]{7}{*}{ sp|P82832 } & \multirow[t]{7}{*}{ Temporin-1LC } & TRINITY_DN2695_C0_g1_i1 & 175.444 \\
\hline & & & TRINITY_DN72771_c0_g1_i1 & 105.843 \\
\hline & & & TRINITY_DN73309_c0_g1_i1 & 140.262 \\
\hline & & & TRINITY_DN80955_c1_g1_i1 & 254.369 \\
\hline & & & TRINITY_DN80955_c1_g2_i1 & 387.789 \\
\hline & & & TRINITY_DN84769_c0_g1_i1 & 373.663 \\
\hline & & & TRINITY_DN86165_c3_g1_i1 & 155.493 \\
\hline & sp|P82881 & Temporin-1Cb & TRINITY_DN56332_C0_g1_i1 & 105.694 \\
\hline & sp|P84858 & Temporin-GH & TRINITY_DN77159_c0_g2_i9 & 102.951 \\
\hline \multirow[t]{9}{*}{ Tigerinin } & \multirow[t]{3}{*}{ sp|P82652 } & \multirow[t]{3}{*}{ Tigerinin-2 } & TRINITY_DN70574_C0_g1_i1 & 242.086 \\
\hline & & & TRINITY_DN71694_C0_g1_i1 & 101.217 \\
\hline & & & TRINITY_DN85395_C7_g6_i1 & 130.962 \\
\hline & sp|COHL42 & Tigerinin-2OS & TRINITY_DN85615_c2_g3_i2 & 125.931 \\
\hline & \multirow[t]{5}{*}{ sp|P82651 } & \multirow[t]{5}{*}{ Tigerinin-1OS } & TRINITY_DN17276_CO_g1_i1 & 245.073 \\
\hline & & & TRINITY_DN43562_C0_g1_i1 & 104.073 \\
\hline & & & TRINITY_DN6203_C0_g1_i1 & 454.682 \\
\hline & & & TRINITY_DN73092_c0_g1_i1 & 248.482 \\
\hline & & & TRINITY_DN85479_c6_g29_i1 & 457.183 \\
\hline \multirow[t]{2}{*}{ Uperolein } & sp|P08612 & Uperolein & TRINITY_DN111770_C0_g1_i1 & 107.555 \\
\hline & & & TRINITY_DN83356_c0_g1_i1 & 424.761 \\
\hline
\end{tabular}


Table $\mathbf{2}$ List of main antimicrobial peptides from transcriptome (Continued)

\begin{tabular}{|c|c|c|c|c|}
\hline AMP families & Accesion number & Identification & ENA Transcript identification & TPM \\
\hline & & & TRINITY_DN83356_c0_g1_i2 & 339.468 \\
\hline & & & TRINITY_DN83356_c0_g1_i3 & 101.171 \\
\hline & & & TRINITY_DN81958_c0_g1_i1 & 277.617 \\
\hline & & & TRINITY_DN81958_C0_g1_i2 & 216.205 \\
\hline \multirow[t]{6}{*}{ Uperin } & sp|P82036 & Uperin-5.1 & TRINITY_DN87247_C0_g1_i1 & 332.281 \\
\hline & & & TRINITY_DN87247_c1_g1_i1 & 137.665 \\
\hline & & & TRINITY_DN87247_c0_g1_i3 & 185.119 \\
\hline & & & TRINITY_DN77327_C0_g2_i1 & 238.182 \\
\hline & & & TRINITY_DN87210_C0_g1_i1 & 105.306 \\
\hline & & & TRINITY_DN87210_C0_g2_i1 & 132.473 \\
\hline
\end{tabular}

CS was capable of degrading azocasein. The tests in presence of EDTA and PMSF did not show differences.

\section{Discussion}

Although toads are widely spread in Brazilian and Latin America territory, its cutaneous peptides and proteins have come to the spotlight only recently and the scarce information hinders its assessment $[11,16,17]$.

The present study reveals the abundance of the $R h i$ nella schneideri mucous gland defense peptides and protein through the RNA-seq transcriptome and some peptides and proteins present in its secretions. We used the cutaneous secretions from one specimen that was milked prior the gland extraction to obtain a more accurate result comparing the biochemical tests with the transcriptome.

Transcriptome survey is snapshots of mRNA obtained in a specific time. Therefore, the first step of this study was milking the toad's skin secretions to maximize the transcripts production. We also used the secretion to carry out biochemical analysis subsequently. Using the secretions from the same toad to perform the experiments carried out in this paper allows us to discard any variation that may occur and obtain a more accurate analysis. RNA-seq analysis revealed the presence of distinct proteins and peptides. Most of the transcripts are related to housekeeping function, as expected, but we found novel proteins in the skin. As frog defense peptides are better characterized, we enriched the functional annotation using DADP database. Using this approach, there were several classes of AMP that are summarized in Table 2. The contigs that presented the highest values of TPM belong to tachykynins, temporin, peroniin and buforin classes. Bufo bufo, $B$. verrucossismus and $B$. variabilis skin secretions act as antimicrobial agent against Escherichia fecalis and E. faecium with lower minimum inhibitory concentration than ampicillin [18].

The molecular classes of AMPs found in this transcriptome are discussed below.

\section{Kassinin and Rugosauperolein}

Kassinin and rugasoperolein AMPS belong to the tachykinin family of neuropeptides. Tachykinins are well present in amphibian secretions and higher organisms, acting as neurotransmitters and neuroprotective agents in the latter. They present a C-terminal conserved region Phe-X-GlyLeu-Met, that is known for this family's activity [32]. Regarding amphibians secretions, kassinin, a dodecapeptide, was the first tachykinin described from Kassina senegalensis methanol skin extract, which shows similar activity as substance $\mathrm{P}$, physalaemin and eldosein, being able to stimulated salivary secretion, act as hypotensive agent and stimulate smooth muscle $[33,34]$. Contig TRINITY_DN127618_c2_g1_i1 is very similar to the first kassinin ever identified.

Rugosauperolein was discovered in Uperoleia rugosa skin methanol extracts and was named after the tachykinin uperolein [35].

Tachykinins are widely studied mainly in higher organisms due their interesting neuroprotective activity. Despite it is known that they are present in amphibian skin, their role as AMP is poorly studied. As their role as neurotransmitters is well established, here we hypothesize they act not as a AMP, but as a host defense molecule that prevents the toad from predation along with micro molecules that are already known to play this role [36]. We use the same hypotheses to the presence of peroniin. Although we cannot discard the possibility of peroniin present any antimicrobial activity, we believe it is produced to prevent $R$. schneideri to be swallowed, once tachykinins are known to show neuroactivity being able to cause gut tissue contraction [37].

\section{Temporin}

Temporins were discovered in a cDNA library from Rana temporaria skin and later obtained in $R$. temporalis' skin secretions. They are effective against gram positive Bacillus megaterium and Escherichia coli with different sensitivities. This AMP family comprises 
Table 3 List of other peptides and protein of interest from the transcriptome

\begin{tabular}{|c|c|c|c|c|}
\hline Protein class & Accession number & Identification & ENA transcript identification & TPM \\
\hline \multirow[t]{2}{*}{ Cobatoxin } & gb|DR51150.1 & Cobatoxin & TRINITY_DN69643_c0_g1_i1 & 94.407 \\
\hline & gb|AKJ54497.1 & Cobatoxin A & TRINITY_DN121110_c0_g1_i1 & 0.40738 \\
\hline \multirow[t]{13}{*}{ Galectin } & gb|KPJ04718.1 & Galectin-4 & TRINITY_DN74940_C0_g1_i1 & 0.372625 \\
\hline & gi| 847,127,031 & Galectin-8 & TRINITY_DN78589_c0_g2_i1 & 149.955 \\
\hline & gi|768,932,680 & Galectin-4-like & TRINITY_DN78656_c0_g1_i1 & 224.999 \\
\hline & \multirow[t]{2}{*}{ gi|692,190,428 } & \multirow[t]{2}{*}{ Galectin 9} & TRINITY_DN80231_C0_g1_i1 & 0.180761 \\
\hline & & & TRINITY_DN80231_c0_g1_i2 & 0.188734 \\
\hline & \multirow[t]{2}{*}{ gi|591,365,832 } & \multirow[t]{2}{*}{ Galectin-9C-like } & TRINITY_DN83688_c1_g1_i1 & 101.232 \\
\hline & & & TRINITY_DN83688_c1_g1_i4 & 170.619 \\
\hline & gi| $928,062,140$ & Galectin-4-like & TRINITY_DN83688_c1_g1_i3 & 0.702454 \\
\hline & gi|512,835,424 & Galectin-12 & TRINITY_DN86454_C0_g1_i1 & 197.028 \\
\hline & \multirow[t]{3}{*}{ gi| $512,835,420$} & \multirow[t]{3}{*}{ Galectin-12 } & TRINITY_DN86454_C0_g1_i2 & 189.779 \\
\hline & & & TRINITY_DN86454_C0_g1_i3 & 0.440812 \\
\hline & & & TRINITY_DN86454_c0_g1_i4 & 0.561889 \\
\hline & gi $2,554,855$ & Galectin A Chain A, & TRINITY_DN92920_c1_g1_i1 & 672.677 \\
\hline \multirow[t]{6}{*}{ Ficolin } & gi|512,864,759 & Ficolin-2-like & TRINITY_DN75601_c0_g1_i1 & 0.832349 \\
\hline & gi|530,624,987 & Veficolin-1-like & TRINITY_DN81241_C1_g5_i1 & 369.808 \\
\hline & gi|611,978,444 & Ficolin-2-like & TRINITY_DN85932_c0_g1_i3 & 104.045 \\
\hline & gi|512,864,763 & Ficolin-2-like & TRINITY_DN86611_c9_g1_i1 & 932.407 \\
\hline & \multirow[t]{2}{*}{ gi| $148,230,483$} & \multirow[t]{2}{*}{ Ficolin } & TRINITY_DN86611_C9_g2_i1 & 120.763 \\
\hline & & & TRINITY_DN86611_c9_g2_i2 & 205.043 \\
\hline \multirow[t]{18}{*}{ Phospholipase $\mathrm{A}_{2}$} & gi|512,862,492 & $\begin{array}{l}\text { Cytosolic Phospholipase } \\
\text { A2 zeta }\end{array}$ & TRINITY_DN47903_c0_g1_i1 & 0.447251 \\
\hline & gi|512,929,364 & $\begin{array}{l}\text { Group XIIA secretory } \\
\text { phospholipase A2 }\end{array}$ & TRINITY_DN86978_c1_g1_i1 & 0.340838 \\
\hline & gi|530,606,756 & $\begin{array}{l}\text { Phospholipase A2, minor } \\
\text { isoenzyme-like }\end{array}$ & TRINITY_DN69641_C0_g1_i1 & 0.724376 \\
\hline & gi|657,561,888 & $\begin{array}{l}\text { Group } 10 \text { secretory } \\
\text { phospholipase A2-like }\end{array}$ & TRINITY_DN71078_c0_g1_i1 & 111.032 \\
\hline & \multirow[t]{2}{*}{ gi|700,363,984 } & \multirow{2}{*}{$\begin{array}{l}\text { Cytosolic phospholipase } \\
\text { A2 gamma }\end{array}$} & TRINITY_DN84494_c4_g4_i1 & 0.294445 \\
\hline & & & TRINITY_DN84494_c4_g5_i1 & 0.141515 \\
\hline & \multirow[t]{2}{*}{ gi|847,119,410 } & \multirow{2}{*}{$\begin{array}{l}85 / 88 \mathrm{kDa} \text { calcium-independent } \\
\text { phospholipase A2 }\end{array}$} & TRINITY_DN79625_c2_g1_i1 & 0.48039 \\
\hline & & & TRINITY_DN79625_c2_g1_i2 & 0.508773 \\
\hline & \multirow[t]{4}{*}{ gi| $884,758,924$} & \multirow{4}{*}{$\begin{array}{l}\text { Cytosolic phospholipase } \\
\text { A2 gamma }\end{array}$} & TRINITY_DN60871_C0_g1_i1 & 0.0554201 \\
\hline & & & TRINITY_DN60871_c0_g2_i1 & 0.273762 \\
\hline & & & TRINITY_DN60871_C0_g3_i1 & 0.081637 \\
\hline & & & TRINITY_DN60871_C0_g4_i1 & 0.278435 \\
\hline & gi| $512,862,492$ & $\begin{array}{l}\text { Cytosolic phospholipase } \\
\text { A2 zeta }\end{array}$ & TRINITY_DN54445_c0_g1_i1 & 162.823 \\
\hline & \multirow[t]{2}{*}{ gi|148,223,595 } & \multirow[t]{2}{*}{ Cytosolic phospholipase A2 } & TRINITY_DN71015_C0_g1_i1 & 107.048 \\
\hline & & & TRINITY_DN71015_c0_g2_i1 & 0.0297482 \\
\hline & gi|48,429,036 & $\begin{array}{l}\text { Phospholipase } A 2 \text { crotoxin } \\
\text { basic subunit } C B C\end{array}$ & TRINITY_DN72825_C0_g1_i1 & 320.644 \\
\hline & gi|129,456 & $\begin{array}{l}\text { Phospholipase A2 homolog } \\
\text { crotoxin acid subunit CA }\end{array}$ & TRINITY_DN72825_c0_g2_i1 & 388.741 \\
\hline & gi|512,834,221 & $\begin{array}{l}\text { Group XV phospholipase } \\
\text { A2 isoform X2 }\end{array}$ & TRINITY_DN74674_c0_g1_i1 & 202.101 \\
\hline
\end{tabular}


Table 3 List of other peptides and protein of interest from the transcriptome (Continued)

\begin{tabular}{|c|c|c|c|c|}
\hline Protein class & Accession number & Identification & ENA transcript identification & TPM \\
\hline & gi|163,916,015 & Phospholipase A2, group XIIA & TRINITY_DN74706_C0_g1_i1 & 250.869 \\
\hline Metallo proteases & gi|148,222,675 & $\begin{array}{l}\text { Disintegrin and metalloproteinase } \\
\text { domain-containing protein } \\
22 \text { precursor }\end{array}$ & TRINITY_DN11900_C0_g1_i1 & 0.414536 \\
\hline \multirow[t]{14}{*}{ Serine proteases } & gb|ACI32835.1 & Serine proteinase-like protein 1 & TRINITY_DN79566_C0_g1_i1 & 0.486137 \\
\hline & gi|913,306,165 & Serine proteinase stubble & TRINITY_DN86427_C0_g1_i1 & 0.326702 \\
\hline & \multirow[t]{4}{*}{ gb|ACI32835.1 } & \multirow[t]{4}{*}{ Serine proteinase-like protein 1} & TRINITY_DN87507_C0_g1_i1 & 17.516 \\
\hline & & & TRINITY_DN87507_c0_g1_i3 & 126.092 \\
\hline & & & TRINITY_DN87507_C0_g1_i4 & 156.004 \\
\hline & & & TRINITY_DN87507_C0_g1_i5 & 0.772103 \\
\hline & \multirow[t]{3}{*}{ gi|380,875,411 } & \multirow{3}{*}{$\begin{array}{l}\text { Thrombin-like Enzyme } \\
\text { gyroxin B1.4 }\end{array}$} & TRINITY_DN87578_C1_g1_i2 & 0.172693 \\
\hline & & & TRINITY_DN87578_c1_g1_i5 & 0.125578 \\
\hline & & & TRINITY_DN87578_C1_g1_i6 & 0.658672 \\
\hline & \multirow[t]{3}{*}{ gb|AEJ32000.1 } & \multirow[t]{3}{*}{ Serine proteinase 6} & TRINITY_DN87578_c1_g1_i3 & 0.266027 \\
\hline & & & TRINITY_DN87578_C1_g1_i4 & 0.246148 \\
\hline & & & TRINITY_DN87578_C1_g1_i8 & 0.29959 \\
\hline & gi| $827,563,139$ & $\begin{array}{l}\text { Serine proteinase-like } \\
\text { protein isoform } X 1\end{array}$ & TRINITY_DN87890_C1_g6_i3 & 0.193263 \\
\hline & gb|AAM69353.1 & Serine proteinase-like protein 2 & TRINITY_DN87890_c1_g6_i5 & 0.508857 \\
\hline
\end{tabular}

anionic, hydrophobic, small peptides (8-17 amino acids) that are folded in alpha-helices and which potency is related to the final net charge [38, 39]. Following its first discovery, temporins were also found in secretions of different species of the genus Euphycits, Limnonectes, Hypsiboas, Amolops, Hylarana and Lithobates [39]. Regarding their biotechnological applications, there are more than 20 deposited patents related to them, which varies from their pharmacological use as anti-HIV to obtaining of transgenic plant resistant to pathogens [39].

Deposited contig TRINITY_DN86407_c15_g2_i1 is similar to temporin-ECa, from Euphlyctis cyanophlyctis, the skittering frog. As other temporins, it shows activity against gram-positive bacteria $E$. coli, K. pneumonia,
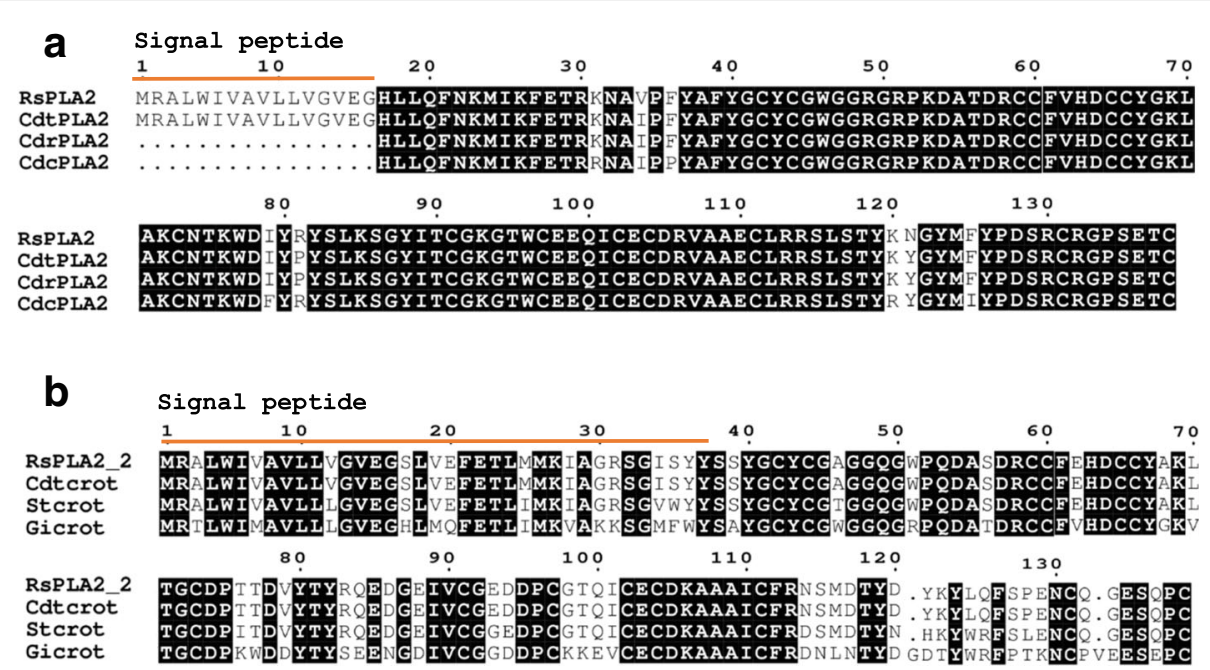

Fig. 1 Sequence alignment of phospholipases $A_{2}$ discovered in this transcriptome (RsPLA $)$ and those available in databases. a Alignment of protein codified by contig TRINITY_DN72825_CO_g1_i2, named RsPLA2 and different basic crotoxins subunits from Crotalus durissus terrificus CdtPLA 2 (PA2B6_CRODO), Crotalus durissus collilineatus CdcPLA 2 (PA2B6_CRODO) and Crotalus durissus ruruima (PA2BA_CRODR). b Alignment of protein codified by contig TRINITY_DN72825_C0_g1_i1, named RsPLA2_2 and different acidic crotoxins subunits, also known as crotapotin, from Crotalus durissus terrificus Cdtcrot (PA1A_CRODU), Sistrurus tergeminus Stcrot (PA2A_SISTE) and Gloydius intermedius Gicrot (A0A096XPP1_GLOIT). Signal peptide is indicated by the orange line above the sequences. Alignment was generated with the aid of Multalin and formatted using Espript 3.0 


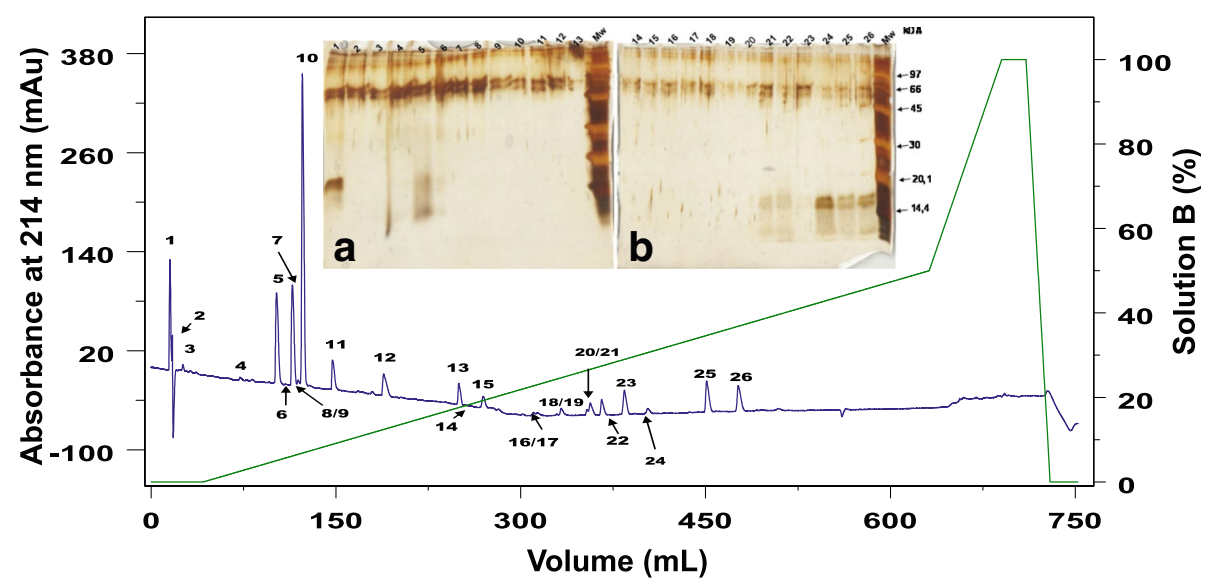

Fig. 2 Cutaneous secretion chromatogram in C18 column RP-FPLC and the SDS-PAGE profile of each fraction. The blue line represents the absorbance monitored at $214 \mathrm{~nm}$ and the green line represents the concentration of solution B. Each fraction was analyzed in SDS-PAGE stained with silver (insert figures). Insert figure a represents fractions CS1 to CS13 and insert figure b shows fractions CS14 to CS26. The wells at left show the low molecular weight marker from GE Healthcare

Micrococcus luteus and Staphylococcus aureus, and low hemolytic activity [40] .

\section{Peroniin}

Peroniins discovery was made through mass spectrometry analysis of Litoria peronii skin secretions in winter and summer. Albeit they are considered AMP, there is no report of their test against neither gram positive nor gram negative bacteria. In fact, peronnins are the major component of $L$. peronii secretions both summer and winter and they possess activity over smooth muscle causing its contraction. There is only one report of peroniin up to date [41].

\section{Buforin}

This AMP family was first discovered in Bufo Bufo gargarizans stomach. This family comprises small peptides (approximately $6.5 \mathrm{kDa}$ ) and are effective against several gram positive and negative bacteria [42]. They are derived from histone $\mathrm{H} 2 \mathrm{~A}$ and belong to the toads' innate immune system. While buforin I is secreted in the stomach protecting the toad against pathogen ingested microorganisms and further binding to the mucosa biofilm enhancing its protection, buforin II does not have its mechanism of action fully elucidated, but it is hypothesized they bind to microorganism's nucleic acid destroying it [43]. In this study, contig TRINITY_DN54614_c0_g1_i1 is related to buforin-EC, isolated from skin secretions of frog Euphlyctis cyanophlyctis, which has shown activity against Staphyloccoccus aureus and Escherichia coli [40]. We believe temporins and buforins, along with other AMPs, act as protection against microorganisms, due to the wellestablished activity of these molecules against gram positive and negative bacteria. The diversity of AMP found in the skin proves that glandular secretions work as a biochemical enriched barrier for the toads' protection.

In addition to the AMPs many other protein components, which probably have relevant roles for frog defense, have been identified in the transcriptome, among them cobatoxins, PLA $\mathrm{P}_{2}$, proteases, ficolin and galectins. These molecular classes are discussed below. Interestingly, ficolin was one of the major contigs found in the transcriptome survey.

\section{Cobatoxin}

Cobatoxins were firstly reported in the Centruroides noxius scorpion poison as potassium channel-blocking toxins, belonging to the $\alpha$-K-toxins, subfamily 9 . They are moderate affinity blockers of $\mathrm{K}^{+}$voltage-dependent channels Shaker and $\mathrm{K}_{\mathrm{v}} 1.1$ [44]. Cobatoxin from Helicoverpa armigera is mostly connected to the insect defense. Its level of expression has significantly raised after infection with both gram positive and negative bacteria [45]. Regarding Spodopetra exigua cobatoxin, gene expression analysis from the insect midgut after the exposition of the insect to B. thuringiensis toxins revealed an increase of the mRNA coding for this protein, indicating that it plays a fundamental role in the insect defense [46]. Furthermore, an analysis of Galleria mellonella challenged against Micrococcus luteus has shown that cobatoxin is likely to maximize the potential of other innate AMPs from the insect [47].

Here, we hypothesize that cobatoxin is also part of the immune system of the toad.

We also found other proteins of interest that are not catalogued as AMP with the aid of non-redundant NCBI database annotation: 

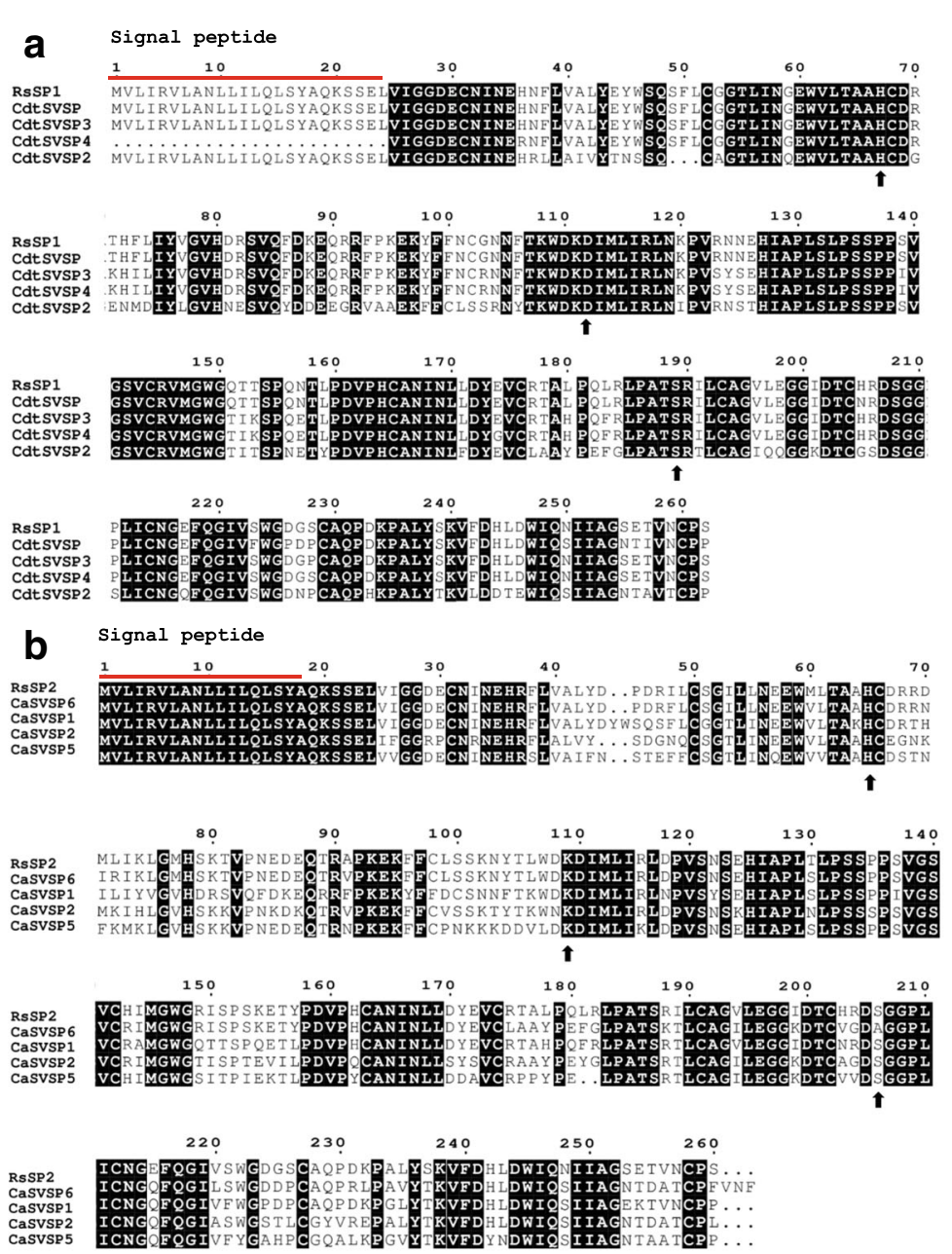

Fig. 3 Sequence alignment of serine protease discovered in this transcriptome (RSSP) and those from different snake venoms (SVSP) available in datadases. a Alignment of protein codified by contigs TRINITY_DN87578_C1_g1_i3, TRINITY_DN87578_C1_g1_i4 and TRINITY_DN87578_C1_g1_i8, named RsSP1 and gyroxin serine proteases from snake venoms (SVSPs) from Crotalus durissus terrificus (CdtSVSP3, VSP13_CRODU), CdtSVSP 2 (VSP21_CRODU) CdtSVSP4 (VSP14_CRODU). b Alignment of serine protease codified by contigs TRINITY_DN87578_C1_g1_i2, TRINITY_DN87578_C1_g1_i5 and TRINITY_DN87578_c1_g1_i6, named RsSP2 and gyroxin SVSP from Crotalus adamanteus 1, CaSVSP1 (VSP1_CROAD), CaSVSP2 (VSP2_CROAD), CaSVSP5 (VSPE_CROAD) and CaSVSP6 (A0A1W7RB84_CROAD). Signal peptide is indicated above the sequences and the arrows beside the alignment indicates the amino acids that are important to the catalytic activity. Alignment was generated with the aid of Multalin and formatted using Espript 3.0

\section{Phospholipase $A_{2}$}

Phospholipase $\mathrm{A}_{2}\left(\mathrm{PLA}_{2}\right)$ catalyze the hydrolysis of phospholipids in the sn2 position releasing arachidonic acid and lysophosphatidic acid, which are precursors of signaling molecules in immune response, inflammation, pain and cell regulatory processes [48-50]. They can be found in different tissues and organelles and are often small proteins $(14-18 \mathrm{kDa})$, and their stability varies according to the number of disulfide bonds. Secreted $\mathrm{PLA}_{2} \mathrm{~S}$ are one of the major components of Elapidae and Viperidae snake venoms [51]. In fact, the crotoxin was the first toxin isolated almost 100 years

Table $4 \mathrm{~N}$-terminal sequences from the poison fractions by Edman's degradation technique

\begin{tabular}{lll}
\hline Fraction & Protein sequence & Protein family \\
\hline CS1 & RWEECEDCDDDQDDQQQQQCAKDDDDE---Q-QQD & Lectin \\
CS24a & GLLEFNKMIKFETRKNAIPFYAFYGCYCGWGGRRRPK & PLA $_{2}$ \\
CS24b & -TPFYKGAAGQQVPQDIVNYYAFGGCQK-EP-PMRY-EY-VNAGGDQ-----D & Galectin $_{\text {CS25 }}$ \\
CS26 & -L-EFNKMIKFETRKNAIPFYAFY & PLA $_{2}$ \\
\hline
\end{tabular}




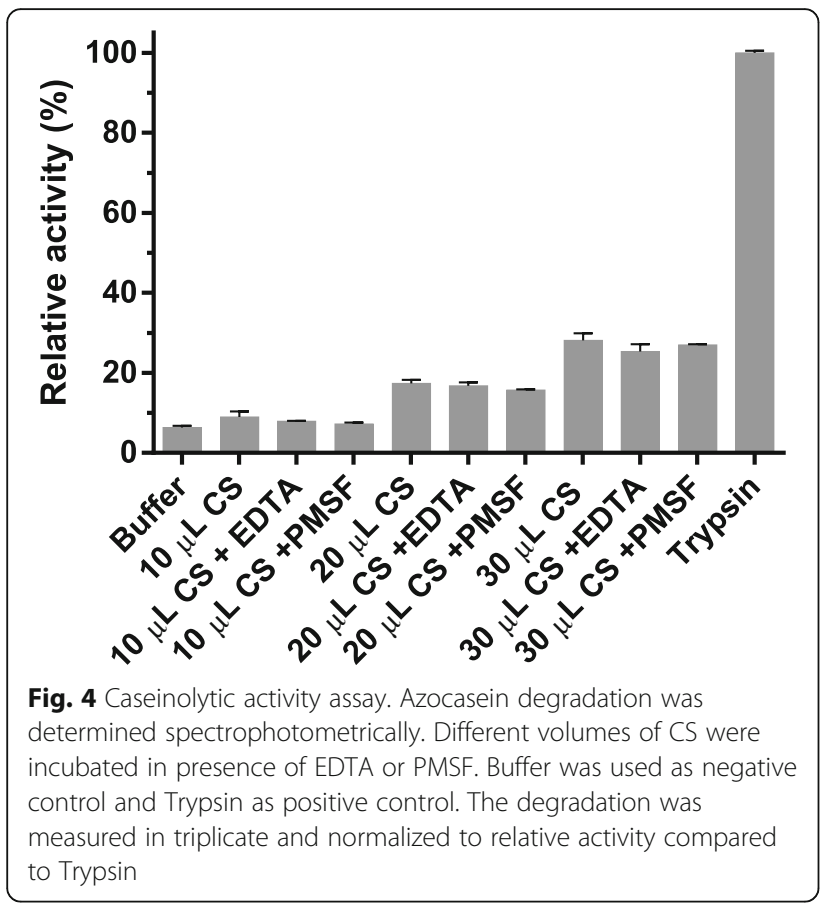

ago and its sequence has been determined for more than 30 years now, and the cloning was successfully obtained in the 80's [52, 53].

In Crotalus snake venoms, crotoxin is composed of two non-covalently bound subunits (one acidic and one basic). The basic component $(\mathrm{CB})$ is a catalytic active $\mathrm{PLA}_{2}$ whereas the acidic component $(\mathrm{CA})$ is a $\mathrm{PLA}_{2}$ catalytically inactive responsible to direct $\mathrm{CB}$ towards specific sites that lead to neurotoxic actions [54-58].

In humans, secreted PLA 2 group IIA can be found in tears and it is most likely to play a defensive role in eyes defense against gram-positive bacteria, but no response to gram-negative bacteria [59]. These molecules are also found in dromedary tears and showed activity against both gram positive and negative bacteria [60]. Thus, a PLA 2 isolated from Daboia Russelli venom was able to strongly inhibit gram negative bacteria and also showed activity against gram positive bacteria isolated from human [61] . Therefore, we also assume the protective action in toad skin.

\section{Serine and metalloproteases}

The contig TRINITY_DN11900_c0_g1_i1 is highly homologous to a metallo-disintegrin protease that has been related to Xenopus laevis reproduction [62] and neural crest development [63]. Although it was possible to obtain a full-length transcript in neural cells, the metallo-disintegrin did not have the catalytic domain, which indicates this protein acts as a transmembrane receptor [63].
Several full-length sequences were related to serine proteases. Three of them containing the same coding sequence (herein named RsSVSP) highly related to gyroxin, a non-lethal serine protease with neurotoxic effects that causes a neurological syndrome in mice known for the animal's movements as rotation to a barrel roll [64]. The contigs TRINITY_DN87578_c1_g1_33, TRINITY_DN87578_c1_g1_i 4 and TRINITY_DN87578_c1_g1_i8 encode a protein, named RsSVSP2, very similar to serine protease 6 from C. adamanteus (gi|338,855,342), also found in a transcriptoma survey [65].

\section{Ficolin and galectin}

Ficolins are a group of oligomeric lectin that present fibrinogen-like and collagen-like domains and possess a carbohydrate binding domain (CRD), being $\mathrm{N}$-acetylglucosamine (GlcNAc) the carbohydrate that presents the major number of galectins specificity $[66,67]$. They are capable of activating complement system via lectin pathway, and aggregate some bacteria enhancing phagocytosis showing their relevant role in organisms' defense [68].

Galectins are lectins which major ligand is $\beta$-galactose-containing glycoconjugates and show their CRD conserved. They may bind to cell-surface and matrix glycans, being able to control intracellular signaling, and protein interactions dependent pathways [69]. Due to its action over neurological system, we believe it also plays a possible role as host defense peptides (HDP) and may provide protections against animal predation adding or even enhancing the symptoms of micro molecules present in parotoid glands that show neurological effects [10].

The contig TRINITY_DN92920_c1_g1_i1 is similar to galectin, an S-type lectin, from Rhinella arenarum ovary, probably playing a developmental regulatory role [70]. In contrast, Хепориs galectins from the animal's skin are believed to act as HDP [71]. Recently, a galectin was found in parotoid secretion from $R$. schneideri using mass spectrometry de novo sequencing [11].

Crude secretion has shown a different profile from parotoid poison presented before $[11,12]$. In the cutaneous secretion (Additional file 2) it is possible state there are a richness of protein content which molecular weight varies from high molecular mass (around $100 \mathrm{kDa}$ ) to low molecular mass (less than $14 \mathrm{kDa}$ ), but there is a strong band with approximately $60 \mathrm{kDa}$ that is common to the profile presented in male and female parotoid secretions from Piaui, Brazil [11]. After analyzing the variation of mass, crude secretion was submitted to RP-FPLC to further investigation.

The RP-FPLC of the secretion resulted in 26 fractions, which were further analyzed by SDS-PAGE. The fractions CS1, CS24-CS26 were submitted to Edman's 
degradation sequencing, because they showed protein bands on SDS-PAGE. Other fractions might contain micro molecules from poison and this method of chromatography was already used for isolation of those molecules from $R$. schneideri parotoid poison before [15].

Fractions CS24, CS25 and CS26 showed sequences regarding a $\mathrm{PLA}_{2}$ from snake venom [72]. Fractions CS24a, CS25 and CS26 contain PLA $\mathrm{P}_{2}$ isoforms similar to a basic PLA $\mathrm{P}_{2}$ isolated from Crotalus durissus terrificus (PA2BF_CRODU). As expected, we were able to predict the protein sequence from the contig TRINITY_DN72825_c0_g1_i1. We also retrieved contigs related to the acidic PLA PL $_{2}$ In crotoxin, one of the major components of Crotalus spp. venom, both units (basic and acidic PLA $\mathrm{A}_{2}$ ) are bonded by a non-covalent bound. The acid subunit (also named crotapotin) is devoid of activity, acting as a stabilizer, and the basic subunit is catalytically active and toxic [52]. Despite its role in the snake venom, we hypothesize this $\mathrm{PLA}_{2}$ is related to toad's immune system, similarly to secreted PLA $\mathrm{A}_{2}$ in human [59] and dromedary tears [60], especially facing the probability of the toad infection by amoeba.

CS24b sequencing also presents a protein that showed similarity to a galectin-1 from Rhinella arenarum (gi|255a855; sp.|P5621). It is a beta-galactosylbinding lectin discovered in toad ovary and similar to the mammalian one that binds to different carbohydrates intra and extra-cellular, probably regulating developmental process in toads oocytes [70]. There are three contigs related to this protein, but none of them is complete. Xenopus laevis is known to secrete a lectin, along with other defense peptides, which plays a role not only in defense against pathogenic microorganisms, but also against predators and another unknown structural role [71].

CS1 sequenced revealed this fraction contains more than one protein; the first putative protein is similar to a C-type lectin isolated from Helicoverpa armigera (gi|385,202,653) that is up regulated in the presence of Escherichia coli injection, indicating it role as a defense protein [45], but it was not found in the transcriptome. CS1 is also similar to the lectin from Naegleria gliberia (gi|290,983,012), an amoeba that can be found in moist habitats and freshwater, that was unrevealed in its genome [73]. We could not retrieve any contigs related to this protein, which indicates a possible contamination of the toad skin by this microorganism. This contamination may explain the high levels of defense peptides and proteins found in the transcriptome and other fractions. It is known that toads might produce toxins that are specific against pathogens [74].

Beyond the AMP assessment and the discovery of other peptides and proteins of defense, we also retrieved some proteases. As the transcriptome showed some evidence of serine and metalloproteases, we decided to carry out an exploratory experiment to survey the presence of these proteins in the poison. Azocasein degradation assay is a classic test to determine the presence of either of these enzymes since proteolytic activity is evaluated. Raw secretion can result in dose dependent azocasein degradation. We used EDTA, which is a chelator agent that inhibits metalloproteases, and PMSF that inhibits serine proteases to investigate which class is most likely to be present in the raw secretion and cause the casein degradation. Despite our best efforts, it was not possible to determine which class of protease was more active, once the controls with PMSF and EDTA did not show any statistical relevance. However, we believe serine protease are the main responsible for this result, since there are more full-length contigs with higher TPM in the transcriptome and only one low expressed (TPM <1) fulllength contig coding for a metalloprotease. In addition, a serine protease was found in Bufo bufo parotoid secretion through a proteomic analysis [17], indicating it may be produced in this toad secretion too, but further characterization is needed.

Here we presented the first transcriptome survey from $R$. schneideri skin. As the results showed, this study paves the way for discovering new molecules besides characterizing an important secretion and the glands where they are produced.

\section{Conclusions}

Although Rhinella schneideri toads are known to possess bioactive molecules in its secretion, it is still poorly studied when compared to other venomous and poisonous animals. Most of the studies comprises the parotoid micro molecules secretions in detriment of cutaneous secretion. This is the first study to use a high throughput RNA-seq technology to investigate $R$. schneideri cutaneous secretions and the first one to focus on defense peptides and proteins. Furthermore, using the milked secretion and skin from the same toad allowed an accurate analysis of protein expression, once individual variation was dismissed. The results obtained herein showed evidence of novel AMP and enzymes that need to be further explored.

\section{Additional files}

Additional file 1: Gene ontology of Rhinella schneideri skin transcriptome. The Gene Ontology is divided in biological proccess, molecular function and cellular component. (DOCX $13 \mathrm{~kb}$ )

Additional file 2: Eletrophoretic profile of Rhinella schneideri's cutaneous secretion. Different volumes $(5,10$ and $20 \mu \mathrm{L})$ of CS were analyzed by $12,5 \%$ SDS-PAGE and stained with Coomasie Blue PhastGel ${ }^{\text {тм }} \mathrm{R}$-350. MW-molecular weight marker; $5 \mu \mathrm{L}-5 \mu \mathrm{L}$ of $\mathrm{CS} ; 10 \mu \mathrm{L}-10 \mu \mathrm{L}$ of CS; $20 \mu \mathrm{L}-20 \mu \mathrm{L}$ of CS. All the samples were reduced in the presence of 
$\beta$-mercaptoethanol and boiled for 10 minutes befora application in the SDS-PAGE. (PPTX $189 \mathrm{~kb}$ )

\section{Abbreviations}

ACN: Acetonitrile; AMP: Antimicrobial peptides; CS: Cutaneous secretion; DADP: Anuran antimicrobial peptides database; DEPC: Diethylpyrocarbonate; EDTA: Ethylenediamine tetraacetic acid; ENA: European Nucleotide Archive; GO: Gene ontology; HDP: Host defense peptides; NCBI: National Center for Biotechnology Information; pident: Protein identity; PLA 2 : Phospholipase $A_{2}$ PMSF: Phenylmethylsulfonyl fluoride; PVDF: Polyvinylidene fluoride; RNAseq: RNA sequencing; RP-FPLC: Reversed phase fast protein liquid chromatography; SDS-PAGE: Denaturing polyacrylamide electrophoresis gel; SVSP: Snake venom serine protease; TCA: Trichloroacetic acid; TFA: Acid trifluoracetic; TPM: Transcripts Per Kilobase Million

\section{Acknowledgements}

The authors are thankful for Karla de Castro Fiqueiredo Bordon, PhD, for the $\mathrm{N}$-terminal sequencing, lara Aimê Cardoso for technical support and biologist Luiz Henrique Anzaloni Pedrosa.

\section{Funding}

The São Paulo Research Foundation (FAPESP; grant n. 2015/18432-0 and scholarships to PYTS n.2014/15644-3, CTC n. 2013/26200-6 and GAW n. 201/ 06170-8) supported this work. This work was supported in part by the Coordination for the Improvement of Higher Education Personnel (CAPES) through Programa Editoração CAPES (edital n. 13/2016, auxílio n. 0722/2017, processo n. 88881.142062/2017-01) and by the National Council for Scientific and Technological Development (CNPq) through Programa Editorial CNPq/ CAPES (process n. 440954/2017-7).

\section{Availability of data and materials}

The datasets generated and/or analyzed during the current study are available in the European Nucleotide Archive- ENA repository, http://www.ebi.ac.uk/ena.. The accession number for the transcriptome is: PRJEB27393 and for the coding sequences mentioned in this paper is: ERZ693103.

\section{Authors' contributions}

PYTS was responsible for carrying out the transcriptome data analysis, toad's poison biochemical analysis and was a major contributor in writing the manuscript. CTC also was responsible for data analysis, design experiment and writing the manuscript. RMF performed the de novo assembling and FPM calculations. GAW performed part of the functional annotation and deposited the data. CUV surpervisioned the in silico analysis and assisted in the project design. ECA coordinated the whole team, assisted in the experiments design and advised PYTS. All authors read and approved the final manuscript.

\section{Ethics approval and consent to participate}

Not applicable.

\section{Consent for publication}

Not applicable.

\section{Competing interests}

The authors declare there are no competing interests.

\section{Publisher's Note}

Springer Nature remains neutral with regard to jurisdictional claims in published maps and institutional affiliations.

\section{Author details}

'Laboratory of Animal Toxins, School of Pharmaceutical Scienes of Ribeirão Preto, University of São Paulo, Avenida do Café s/n, Ribeirão Preto, SP, Brazil. ${ }^{2}$ Laboratory of genetics - LABGEN, Institute of Genetics and Biochemistry, Campus Umuarama, Federal University of Uberlândia, Avenida Pará, Uberlândia, MG 1720, Brazil. Department of Physics and Chemistry, University of São Paulo, School of Pharmaceutical Sciences of Ribeirão Preto, Av. do Café s/n, Monte Alegre, Ribeirão Preto, SP 14040-903, Brazil.
Received: 4 July 2018 Accepted: 7 November 2018

Published online: 29 November 2018

\section{References}

1. Koehn FE, Carter GT. The evolving role of natural products in drug discovery. Nat Rev Drug Discov. 2005;4(3):206-20.

2. Newman DJ, Cragg GM, Snader KM. The influence of natural products upon drug discovery. Nat Prod Rep. 2000;17(3):215-34

3. Casewell NR, Wüster W, Vonk FJ, Harrison RA, Fry BG. Complex cocktails: the evolutionary novelty of venoms. Trends Ecol Evol. 2012;28(4):219-29.

4. Fry BG, Roelants $K$, Champagne DE, Scheib $H$, Tyndall JD, King GF, et al. The toxicogenomic multiverse: convergent recruitment of proteins into animal venoms. Annu Rev Genomics Hum Genet. 2009:10:483-511.

5. Chan WY, Ng TB, Yeung HW. Examination for toxicity of a Chinese drug, the toad glandular secretory product chan su, in pregnant mice and embryos. Biol Neonate. 1995;67(5):376-80.

6. Frost DR. Amphibian Species of the World: an Online Reference. vol. 6.0 2018. http://research.amnh.org/vz/herpetology/amphibia/

7. Regis-Alves E, Jared SGS, Maurício B, Mailho-Fontana PL, Antoniazzi MM, Fleury-Curado MC, et al. Structural cutaneous adaptations for defense in toad (Rhinella icterica) parotoid macroglands. Toxicon. 2017;137:128-34.

8. Jared C, Antoniazzi MM, Jordao AE, Silva JR, Greven H, Rodrigues MT. Parotoid macroglands in toad (Rhinella jimi): their structure and functioning in passive defence. Toxicon. 2009;54(3):197-207.

9. Clarke BT. The natural history of amphibian skin secretions, their normal functioning and potential medical applications. Biol Rev Camb Philos Soc. 1997;72(3):365-79.

10. Daly JW, Spande TF, Garraffo HM. Alkaloids from amphibian skin: a tabulation of over eight-hundred compounds. J Nat Prod. 2005;68(10):1556-75.

11. Sousa-Filho LM, Freitas CD, Lobo MD, Monteiro-Moreira AC, Silva RO, Santana LA, et al. Biochemical profile, biological activities, and toxic effects of proteins in the Rhinella schneideri parotoid gland secretion. J Exp Zool A Ecol Genet Physiol. 2016;325(8):511-23.

12. Sciani JM, Angeli CB, Antoniazzi MM, Jared C, Pimenta DC. Differences and similarities among parotoid macrogland secretions in South American toads: a preliminary biochemical delineation. Sci World J. 2013;2013:ID 937407.

13. Schmeda-Hirschmann G, Quispe C, Theoduloz C, de Sousa PT Jr, Parizotto C. Antiproliferative activity and new argininyl bufadienolide esters from the "cururu" toad Rhinella (Bufo) schneideri. J Ethnopharmacol. 2014;155(2):1076-85.

14. Anjolette FA, Leite FP, Bordon KC, Azzolini AE, Pereira JC, Pereira-Crott LS, et al. Biological characterization of compounds from Rhinella schneideri poison that act on the complement system. J Venom Anim Toxins incl Trop Dis. 2015;21:25. https://doi.org/10.1186/s40409-015-0024-9.

15. Shibao PY, Anjolette FA, Lopes NP, Arantes EC. First serine protease inhibitor isolated from Rhinella schneideri poison. J Venom Anim Toxins incl Trop Dis. 2015;21:30. https://doi.org/10.1186/s40409-015-0029-4.

16. Freitas CDT, Sousa-Filho LM, Lima MSCS, Pereira ACTC, Ferreira GP, Lopes FES, et al. Proteins from the Rhinella schneideri parotoid gland secretion exhibit anti-nociceptive effect against nociception induced by inflammation. Biomed Pharmacother. 2017;93:705-8

17. Kowalski K, Marciniak P, Rosinski G, Rychlik L. Toxic activity and protein identification from the parotoid gland secretion of the common toad Bufo bufo. Comp Biochem Physiol C Toxicol Pharmacol. 2018;205:43-52.

18. Nalbantsoy A, Karis M, Yalcin HT, Göcmen B. Biological activities of skin and parotoid gland secretions of bufonid toads (Bufo bufo, Bufo verrucosissimus and Bufotes variabilis) from Turkey. Biomed Pharmacother. 2016;80:298-303.

19. Konig E, Bininda-Emonds OR, Shaw C. The diversity and evolution of anuran skin peptides. Peptides. 2014;63:96-117.

20. Boldrini-Franca J, Cologna CT, Pucca MB, Bordon KC, Amorim FG, Anjolette FA, et al. Minor snake venom proteins: structure, function and potential applications. Biochim Biophys Acta Gen Subj. 2016;1861(4):824-38.

21. Huang L, Li J, Anboukaria H, Luo Z, Zhao M, Wu H. Comparative transcriptome analyses of seven anurans reveal functions and adaptations of amphibian skin. Sci Rep. 2016;6:24069.

22. Yang W, Qi Y, Fu J. Genetic signals of high-altitude adaptation in amphibians: a comparative transcriptome analysis. BMC Genet. 2016;17:134.

23. Zhao F, Yan C, Wang X, Yang Y, Wang G, Lee W, et al. Comprehensive transcriptome profiling and functional analysis of the frog (Bombina maxima) immune system. DNA Res. 2014;21(1):1-13. 
24. Andrews S. FastQC: a quality control tool for high throughput sequence data; 2010.

25. Chen TW, Gan RC, Fang YK, Chien KY, Liao WC, Chen CC, et al. FunctionAnnotator, a versatile and efficient web tool for non-model organism annotation. Sci Rep. 2017;7:10430.

26. Novković M, Simunić J, Bojović V, Tossi A, Juretić D. DADP: the database of anuran defense peptides. Bioinformatics. 2012;28(10):1406-7.

27. Schägger $H$, von Jagow $G$. Tricine-sodium dodecyl sulfate-polyacrylamide gel electrophoresis for the separation of proteins in the range from 1 to 100 kDa. Anal Biochem. 1987;166(2):368-79.

28. Edman P, Begg G. A protein sequenator. Eur J Biochem. 1967;1(1):80-91.

29. Corpet F. Multiple sequence alignment with hierarchical clustering. Nucleic Acids Res. 1988;16(22):10881-90.

30. Robert $X$, Gouet P. Deciphering key features in protein structures with the new ENDscript server. Nucleic Acids Res. 2014;42:W320-4

31. Wang WJ, Shih $\mathrm{CH}$, Huang TF. A novel P-I class metalloproteinase with broad substrate-cleaving activity, agkislysin, from Agkistrodon acutus venom. Biochem Biophys Res Commun. 2004;324(1):224-30.

32. Severini C, Improta G, Falconieri-Erspamer G, Salvadori S, Erspamer V. The tachykinin peptide family. Pharmacol Rev. 2002;54(2):285-322.

33. Anastasi A, Montecucchi P, Erspamer V, Visser J. Amino acid composition and sequence of kassinin, a tachykinin dodecapeptide from the skin of the African frog Kassina senegalensis. Experientia. 1977;33(7):857-8.

34. Erspamer GF, Erspamer V, Piccinelli D. Parallel bioassay of physalaemin and kassinin, a tachykinin dodecapeptide from the skin of the African frog Kassina senegalensis. Naunyn Schmiedeberg's Arch Pharmacol. 1980;311(1):61-5.

35. Nakajima T, Yasuhara T, Erspamer V, Erspamer GF, Negri L, Endean R. Physalaemin- and bombesin-like peptides in the skin of the Australian leptodactylid frog Uperoleia rugosa. Chem Pharm Bull (Tokyo). 1980;28(3): 689-95

36. Sakate M, Oliveira PCL. Toad envenoming in dogs: effects and treatment. J Venom Anim Toxins. 2000;6(1):52-62 www.scielo.br/scielo.php?script=sci_ arttext\&pid=S0104-79302000000100003.

37. Carter MS, Krause JE. Structure, expression, and some regulatory mechanisms of the rat preprotachykinin gene encoding substance $P$, neurokinin a, neuropeptide K, and neuropeptide gamma. J Neurosci. 1990; 10(7):2203-14

38. Simmaco M, Mignogna G, Canofeni S, Miele R, Mangoni ML, Barra D. Temporins, antimicrobial peptides from the European red frog Rana temporaria. Eur J Biochem. 1996:242(3):788-92.

39. Ladram A, Nicolas P. Antimicrobial peptides from frog skin: biodiversity and therapeutic promises. Front Biosci (Landmark Ed). 2016;21:1341-71.

40. Asoodeh A, Azam AG, Chamani J. Identification and characterization of novel antibacterial peptides from skin secretions of Euphlyctis cyanophlyctis. Int J Pept Res Ther. 2012;18(2):107-15.

41. Bilusich D, Jackway RJ, Musgrave IF, Tyler MJ, Bowie JH. The host-defence skin peptide profiles of Peron's tree frog Litoria peronii in winter and summer. Sequence determination by electrospray mass spectrometry and activities of the peptides. Rapid Commun Mass Spectrom. 2009;23(17):2628-36.

42. Park CB, Kim MS, Kim SC. A novel antimicrobial peptide from Bufo bufo gargarizans. Biochem Biophys Res Commun. 1996;218(1):408-13.

43. Cho JH, Sung BH, Kim SC. Buforins: histone H2A-derived antimicrobial peptides from toad stomach. Biochim Biophys Acta. 2008;1788(8):1564-9.

44. Selisko B, Garcia C, Becerril B, Gomez-Lagunas F, Garay C, Possani LD. Cobatoxins 1 and 2 from Centruroides noxius Hoffmann constitute a subfamily of potassium-channel-blocking scorpion toxins. Eur J Biochem. 1998:254(3):468-79.

45. Wang Q, Liu Y, He HJ, Zhao XF, Wang JX. Immune responses of Helicoverpa armigera to different kinds of pathogens. BMC Immunol. 2010;11:9.

46. Crava CM, Jakubowska AK, Escriche B, Herrero S, Bel Y. Dissimilar regulation of antimicrobial proteins in the midgut of Spodoptera exigua larvae challenged with Bacillus thuringiensis toxins or baculovirus. PLoS One. 2015; 10(5):e0125991.

47. Bolouri Moghaddam MR, Tonk M, Schreiber C, Salzig D, Czermak P, Vilcinskas A, et al. The potential of the Galleria mellonella innate immune system is maximized by the co-presentation of diverse antimicrobial peptides. Biol Chem. 2016;397(9):939-45.

48. Narumiya S, Sugimoto Y, Ushikubi F. Prostanoid receptors: structures, properties, and functions. Physiol Rev. 1999;79(4):1193-226.
49. Lin ME, Herr DR, Chun J. Lysophosphatidic acid (LPA) receptors: signaling properties and disease relevance. Prostaglandins Other Lipid Mediat. 2010;91(3-4):130-8.

50. Murakami M, Kudo I. Phospholipase A2. J Biochem. 2002;131(3):285-92.

51. Zambelli VO, Picolo G, Fernandes CAH, Fontes MRM, Cury Y. Secreted phospholipases A2 from animal venoms in pain and analgesia. Toxins (Basel). 2017;9(12):406.

52. Aird SD, Kaiser II, Lewis RV, Kruggel WG. A complete amino acid sequence for the basic subunit of crotoxin. Arch Biochem Biophys. 1986;249(2):296-300.

53. Bouchier C, Ducancel F, Guignery-Frelat G, Bon C, Boulain JC, Menez A. Cloning and sequencing of CDNAs encoding the two subunits of Crotoxin. Nucleic Acids Res. 1988;16(18):9050

54. Bon C. Synergism of the two subunits of crotoxin. Toxicon. 1982;20(1):105-9.

55. Bon C, Changeux JP, Jeng TW, Fraenkel-Conrat H. Postsynaptic effects of crotoxin and of its isolated subunits. Eur J Biochem. 1979;99(3):471-81.

56. Canziani G, Seki C, Vidal JC. The mechanism of inhibition of phospholipase activity of crotoxin B by crotoxin A. Toxicon. 1983;21(5):663-74.

57. Habermann E, Walsch P, Breithaupt H. Biochemistry and pharmacology of the cortoxin complex. II. Possible interrelationships between toxicity and organ distribution of phospholipase a, crotapotin and their combination. Naunyn Schmiedebergs Arch Pharmacol. 1972;273(4):313-30.

58. Rübsamen K, Breithaupt H, Habermann E. Biochemistry and pharmacology of the crotoxin complex. I. Subfractionation and recombination of the crotoxin complex. Naunyn Schmiedebergs Arch Pharmakol. 1971;270(3):274-88.

59. Qu XD, Lehrer RI. Secretory phospholipase A2 is the principal bactericide for staphylococci and other gram-positive bacteria in human tears. Infect Immun. 1998;66(6):2791-7.

60. Ben Bacha A, Abid I. Secretory phospholipase A2 in dromedary tears: a host defense against staphylococci and other gram-positive bacteria. Appl Biochem Biotechnol. 2013;169(6):1858-69.

61. Sudharshan S, Dhananjaya BL. Antibacterial potential of a basic phospholipase A2 (VRV-PL-VIIla) from Daboia russelii pulchella (Russell's viper) venom. J Venom Anim Toxins incl Trop Dis. 2015;21:17. https://doi.org/10. 1186/s40409-015-0014-y.

62. Shilling FM, Krätzschmar J, Cai H, Weskamp G, Gayko U, Leibow J, et al. Identification of metalloprotease/disintegrins in Xenopus laevis testis with a potential role in fertilization. Dev Biol. 1997;186(2):155-64.

63. Cai H, Krätzschmar J, Alfandari D, Hunnicutt G, Blobel CP. Neural crestspecific and general expression of distinct metalloprotease-disintegrins in early Xenopus laevis development. Dev Biol. 1999;204(2):508-24.

64. Alexander G, Grothusen J, Zepeda H, Schwartzman RJ. Gyroxin, a toxin from the venom of Crotalus durissus terrificus, is a thrombin-like enzyme. Toxicon. 1988;26(10):953-60.

65. Rokyta DR, Wray KP, Lemmon AR, Lemmon EM, Caudle SB. A highthroughput venom-gland transcriptome for the eastern diamondback rattlesnake (Crotalus adamanteus) and evidence for pervasive positive selection across toxin classes. Toxicon. 2011;57(5):657-71.

66. Matsushita M. Ficolins in complement activation. Mol Immunol. 2013;55(1):22-6.

67. Stryer JMB, John LT, Stryer L. Lectins are specific carbohydrate-binding proteins; 2002.

68. Matsushita M. Ficolins: complement-activating lectins involved in innate immunity. J Innate Immun. 2010;2(1):24-32.

69. Yang RY, Rabinovich GA, Liu FT. Galectins: structure, function and therapeutic potential. Expert Rev Mol Med. 2008;10:e17.

70. Bianchet MA, Ahmed H, Vasta GR, Amzel LM. Soluble beta-galactosylbinding lectin (galectin) from toad ovary: crystallographic studies of two protein-sugar complexes. Proteins. 2000;40(3):378-88.

71. Marschal P, Herrmann J, Leffler H, Barondes SH, Cooper DN. Sequence and specificity of a soluble lactose-binding lectin from Xenopus laevis skin. J Biol Chem. 1992;267(18):12942-9.

72. Hernandez-Oliveira S, Toyama MH, Toyama DO, Marangoni S, Hyslop S, Rodrigues-Simioni L. Biochemical, pharmacological and structural characterization of a new PLA2 from Crotalus durissus terrificus (south American rattlesnake) venom. Protein J. 2005;24(4):233-42.

73. Fritz-Laylin LK, Prochnik SE, Ginger ML, Dacks JB, Carpenter ML, Field MC, et al. The genome of Naegleria gruberi illuminates early eukaryotic versatility. Cell. 2010;140(5):631-42.

74. Sun T, Zhan B, Gao Y. A novel cathelicidin from Bufo bufo gargarizans Cantor showed specific activity to its habitat bacteria. Gene. 2015;571(2):172-7. 